\title{
On Measurement and Signal Processing of Low Frequent Magnetic Fields
}

\author{
Christian Rückerl \\ Forschungs- und Transferzentrum Leipzig e.V. \\ Wächterstraße 13 \\ 04107 Leipzig
}

\section{Introduction}

The aim of this article is to facilitate automatic treatment of three-dimensional measurement data. Especially the measurement of low frequent magnetic fields with an isotropic field probe can be seen as an example for higher dimensional sensor data.

Magnetic fields are measured and judged according to their possible negative influences on the human body. For a meaningful evaluation of non-sinusoidal fields some methods are applied in frequency domain [1], others on estimation of time derivative or the absolute change of magnetic flux density, which are responsible for induced body currents ([2],[3]). These are restricted by norms and guidelines [4]. Induced body currents can easily be calculated with measured field data of magnetic flux density and a plain loop model of human body. Therefore, a one-dimensional time series is required. The threedimensional time series of measured data has to be transformed into a one-dimensional one. If only one source is assumed, this can easily be realized with a special signal processing after or during measurement which is based on the eigenvalues of the sample covariance matrix. If there are two ore more sources superimposing the fields can be separated with automatic procedures. The use of blind source separation algotithms like Independent Component Analysis is one of the possible alternatives. The introduced methods enable the automatic detection of superimposed fields which can be implemented in measurement equipment. This feature can help the user to valuate the field situation on the spot (e.g. in a working area). The introduced methods will be applicated to measurement data in two examples.

\section{Theory and Methods}

The measuring of a broadband magnetic field by means of a three-dimensional field probe is assumed. The time discrete data of such a measurement can be depicted as a logic record. If every measured vector of magnetic flux density is time accordingly written into each column of a matrix, the data which encompasses $N$ samples, the matrix of measured data will result in (1).

$$
\mathbf{B}=\left(\begin{array}{llll}
B_{x}(1) & B_{x}(2) & \ldots & B_{x}(N) \\
B_{y}(1) & B_{y}(2) & \ldots & B_{y}(N) \\
B_{z}(1) & B_{z}(2) & \ldots & B_{z}(N)
\end{array}\right)
$$

This matrix is the basis of further considerations. To calculate a one-dimensional time series from matrix (1) the equation (2) is often suggested which estimates the absolute value of the field vector for every point in time.

$$
B(n)=\sqrt{B_{x}^{2}(n)+B_{y}^{2}(n)+B_{z}^{2}(n)}
$$

This equation can be used for all $N$ samples of the measurement. The time series $B(n)$ of absolute values is one-dimensional. The resulting time series has, however a deviant frequency spectrum and adifferent shape in time domain. Since the evaluation of magnetic fields takes place in the time domain or in frequency domain the equation (2) is not siutable. It is, therefore, necessary to calculate a onedimensional time series which is usable for exposition evaluation. One solution of this problem is a linear transfomation in measurement space as a rotation of one axis of the coordinate system on the field vector. This operation can be performed with a double rotation (Fig. 1). 


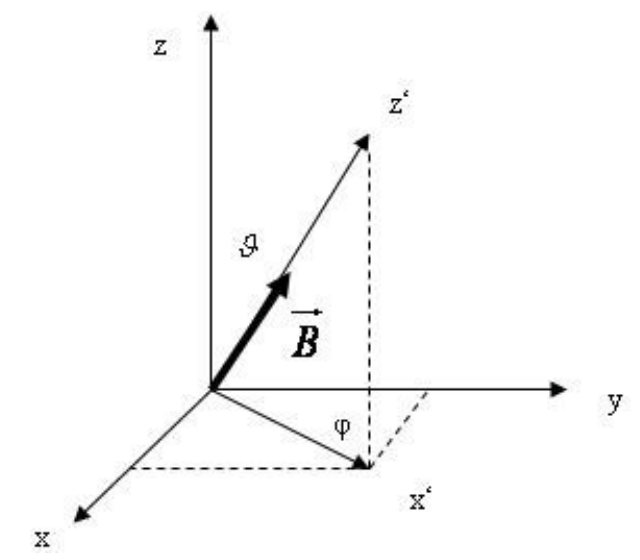

Fig. 1 Field vector in reference measurement coordinate system

A first rotation around the z-axis can be performed with matrix equation (3).

$$
\left(\begin{array}{l}
B_{x}^{\prime} \\
B_{y}^{\prime} \\
B_{z}
\end{array}\right)=\left(\begin{array}{ccc}
\cos \varphi & \sin \varphi & 0 \\
-\sin \varphi & \cos \varphi & 0 \\
0 & 0 & 1
\end{array}\right) \cdot\left(\begin{array}{l}
B_{x} \\
B_{y} \\
B_{z}
\end{array}\right)
$$

A second rotation around the new y'-axis can be calculated with equation (4).

$$
\left(\begin{array}{l}
B_{x}^{\prime \prime} \\
B_{y}^{\prime} \\
B_{z}^{\prime}
\end{array}\right)=\left(\begin{array}{ccc}
\cos \vartheta & 0 & -\sin \vartheta \\
0 & 1 & 0 \\
\sin \vartheta & 0 & \cos \vartheta
\end{array}\right) \cdot\left(\begin{array}{l}
B_{x}^{\prime} \\
B_{y}^{\prime} \\
B_{z}
\end{array}\right)
$$

This two rotations of the z-axis on the field vector can be performed as one matrix operation (5).

$$
\left(\begin{array}{l}
B_{x}^{\prime \prime} \\
B_{y}^{\prime} \\
B_{z}^{\prime}
\end{array}\right)=\left(\begin{array}{ccc}
\cos \vartheta \cdot \cos \varphi & \cos \vartheta \cdot \sin \varphi & -\sin \vartheta \\
-\sin \varphi & \cos \varphi & 0 \\
\sin \vartheta \cdot \cos \varphi & \sin \vartheta \cdot \sin \varphi & \cos \vartheta
\end{array}\right) \cdot\left(\begin{array}{c}
B_{x} \\
B_{y} \\
B_{z}
\end{array}\right)
$$

The matrix elements (trigonometric functions of $\vartheta$ and $\varphi$ ) are calculated from one point in time of measured data. This entails two diadvantages:

- The processing which is applicated on the whole measurement data is based on data at one point in time. An adverse point in a signal with a small signal to noise ratio leads to a ill solution. Practically, one has to estimate this point in time manually.

- This operation does only work when the field of one source in a certain position is measured.

If a superimposition of two or more field sources is measured this operation does not work. In this case the superimposed fields have to be separated. In case $s$ field sources are superimposed the resulting field vector and thus each column of the matrix $\mathbf{B}_{\mathrm{m}}$ can be depicted as a vectorial addition of $s$ components in each (e.g. the $n$-th) time step.

$$
B_{r e s}(n)=B_{1}(n)+B_{2}(n)+\ldots+B_{s}(n)
$$

Assuming that not only the position of the field sources, but also the scanning position is stable, the superimposition for each source can be factorised into a product (7).

$$
B=v_{1} \cdot b_{1}+v_{2} \cdot b_{2}+\ldots+v_{s} \cdot b_{s}
$$


Each unit vector $v_{i}$ contains data concerning the direction of the field vector of the $i$-th source. Each coefficient $b_{i}$, on the other hand, contains data concerning the absolute value and the algebraic signs in the shape of a time series. Equation (7) can be written as a general matrix equation, and as signal model (8) where $\mathbf{A}$ is the mixing matrix and $\mathbf{B}_{\mathbf{s}}$ is the source matrix.

$$
\mathbf{B}_{m}=\mathbf{A} \cdot \mathbf{B}_{s}
$$

$\mathbf{B}_{\mathrm{m}}$ : matrix of measured data

A: mixing matrix

Bs: matrix of one-dimensional time series

Thus one-dimensional time series evolve from the factors $\mathbf{b}_{i}$ in (7) which form the rows of the matrix $\mathbf{B}_{\mathbf{s}}$. This matrix is transformed by the mixing matrix $\mathbf{A}$ on the measured data $\mathbf{B}_{\mathrm{m}}$. $\mathbf{A}$ as well as $\mathbf{B}_{\mathrm{s}}$ are generally unknown. The number of sources $s$ is, however restricted to three for the assumption that a threedimensional measuring in only one spatial measurement position took place. This restriction can be broadened by introducing several more field probes in space

By analysing the sample covariance matrix $\hat{\mathbf{C}}$ one can estimate the number of uncorrelated sources. If the applied sensors are induction coils there is no average value in the three axis signals and thus the expected value is zero. The sample covariance matrix results in (9).

$$
\hat{\mathbf{C}}=\frac{1}{N} \mathbf{B}_{m} \cdot \mathbf{B}_{m}^{\mathbf{T}}
$$

Thereby, the sample covariance matrix consists of covariance of source signals and noise. If noise is negligible the number of eigenvalues different from zero is the number of uncorrelated field sources. In all measurements there are measurement errors and noise, thus the noise covariance matrix and the resulting eigenvalues have to be analysed.

If eigenvalues of $\hat{\mathbf{C}}$ are compared with each other, a simple estimation of uncorrelated sources is possible. There are other methods to estimate the number of sources using information criteria ([5], [6]). If one source is assumed a transformation to obtain a one dimensional time series can be calculated with the eigenvector $\mathbf{d}$ to the (theoretical one) eigenvalue different from zero.

$$
B(n)=\mathbf{d}^{\mathbf{T}} \cdot \mathbf{B}_{m}
$$

Thus a method to calculate a one-dimensional time series from three-dimensional measurement data exists which can be implemented in a software for further field evaluation.

If the analysis of the covariance matrix shows a superimposition of field sources a separation is desired. On the one hand can a separation with known column vectors of matrix $\mathbf{A}$ be performed, on the other hand a blind source separation can be performed with special assumptions to the time series in the matrix $\mathbf{B}_{\text {s. }}$.

If matrix A with its independent column vectors in equation (8) is known, and the influence of errors and noise is negligible, the inversion can be depicted by means of equation (11).

$$
\mathbf{B}_{s}=\left(\mathbf{A} \cdot \mathbf{A}^{\mathbf{T}}\right)^{-1} \mathbf{A}^{\mathbf{T}} \cdot \mathbf{B}_{m}
$$

Such a division can only be applied if the direction vectors in A can be determined, e.g. by a calibrational measuring. It is also possible to calculate them out of the measurement data $\mathbf{B}_{m}$ if periods in which only one source is active can be detected within time of measurement. Submatrices of the measuring data matrix can help finding these periods by applying the analysis on the eigenvalues. The direction vectors of the fields and thus the column vector of $\mathbf{A}$ can be calculated out of these periods. A further advantage results from a spatial filtering facility. Decomposition (11) can be used to eliminate the fields of disturbing sources (e.g. resulting from line currents), especially when EMC measurements are performed. A further method is the decomposition with Independent Component Analysis (ICA). By means of ICA the mixture of signals measured by several sensors can be separated ([7],[8]). An example for the special case of three-dimensional measurement data of magnetic fields can be found in [9]. One assumption of the method is the independence of the source signals (superimposed fields). The precondition of statistic 
independence is fulfilled e.g. by the superimposition of the pulse-shaped field of a welding device and the harmonic alternating field of a line current. The method proceeds as follows:

- Centering (set average value to zero) and whitening (set variance to one) with PCA.

- A contrast criterion (e.g. kurtosis) is being set up to determine the statistic independence of the PCA components.

- Solution of the optimisation task: Maximum statistic independence (minimum kurtosis!).

- Recharge to source time series through an adjustment calculus (mean value and variance).

Since the PCA is an element of the method it also contains the analysis of eigenvalues. It is thus possible to conduct a division of the superimposed field components. The application on several sinusoidal fields, however, will not work. It results in an orthogonal pair of functions which does not allow an inversion to the source components without further pieces of information.

\section{Experiments}

\subsection{Number of sources and one-diomensional time series}

The data of a three-dimesional measurement (Fig. 2) have to be transformed into a one-dimensional time series.
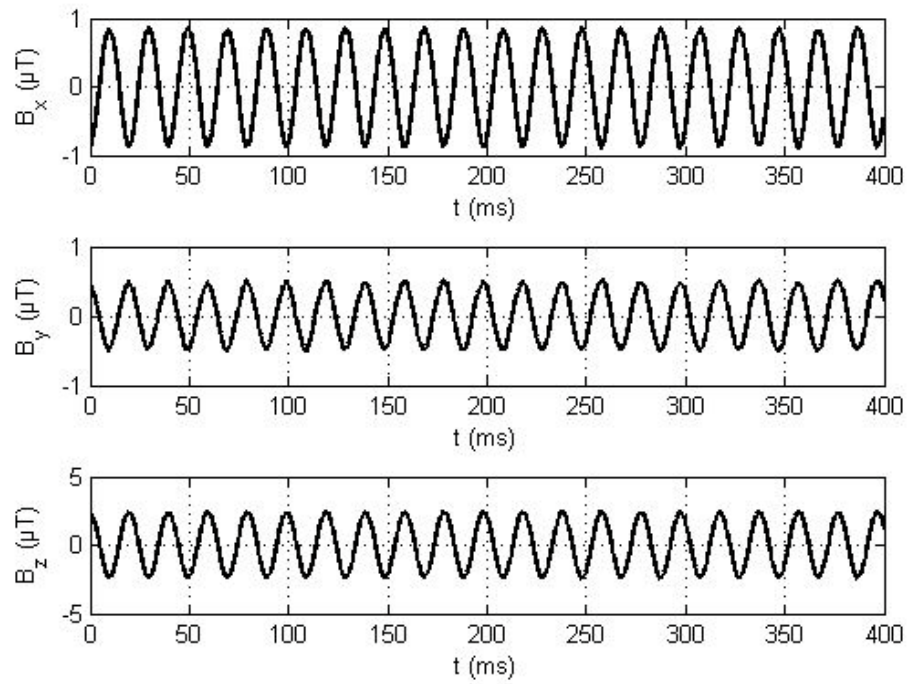

Fig. 2 Data of a three-dimensional measurement of a magnetic field

Thus eigenvalues have to be calculated firstly. With equation (9) and $N=2000$ samples eigenvalues result in $\lambda_{1}=0.0003, \lambda_{2}=0.0017$ and $\lambda_{3}=3.3731$.

From analysis of eigenvalues it can be assumed that there is one source. It is possible to use the $\mathbf{d}_{3}$ eigenvector for a calculation with equation (12). All three eigenvectors are, in this example, put into a matrix $D$ to demonstrate the effect of projecting the measured data on the eigenvectors. The projection results in equation (12).

$$
\mathbf{B}_{123}=\mathbf{D}^{\mathbf{T}} \cdot \mathbf{B}_{m}
$$

The rows of $\mathbf{B}_{123}$ as the result of equation (12) are shown in Fig. 3. 

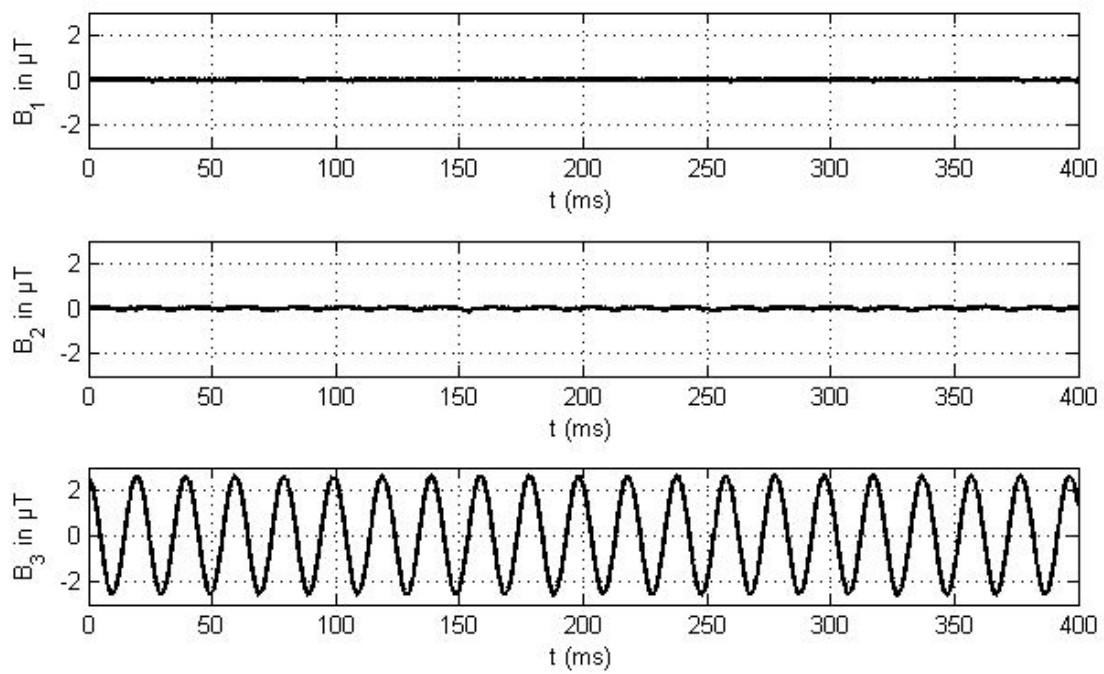

Fig. 3 Projections on the three eigenvectors calculated with equation (12).

It is obvious that nearly the whole variance of measured data is put in the third row $\left(B_{3}\right)$. Thus a projection on the eigenvector $d_{3}$ (belonging to the third/ highest eigenvalue) is the desired operation to estimate a one-dimensional time series.

\subsection{Separation of two superimposed field sources with Independent Component Analysis}

ICA is applied in this example to separate the magnetic fields of a welding device and a line current. Measured data in the three axis are shown in Fig. 4.
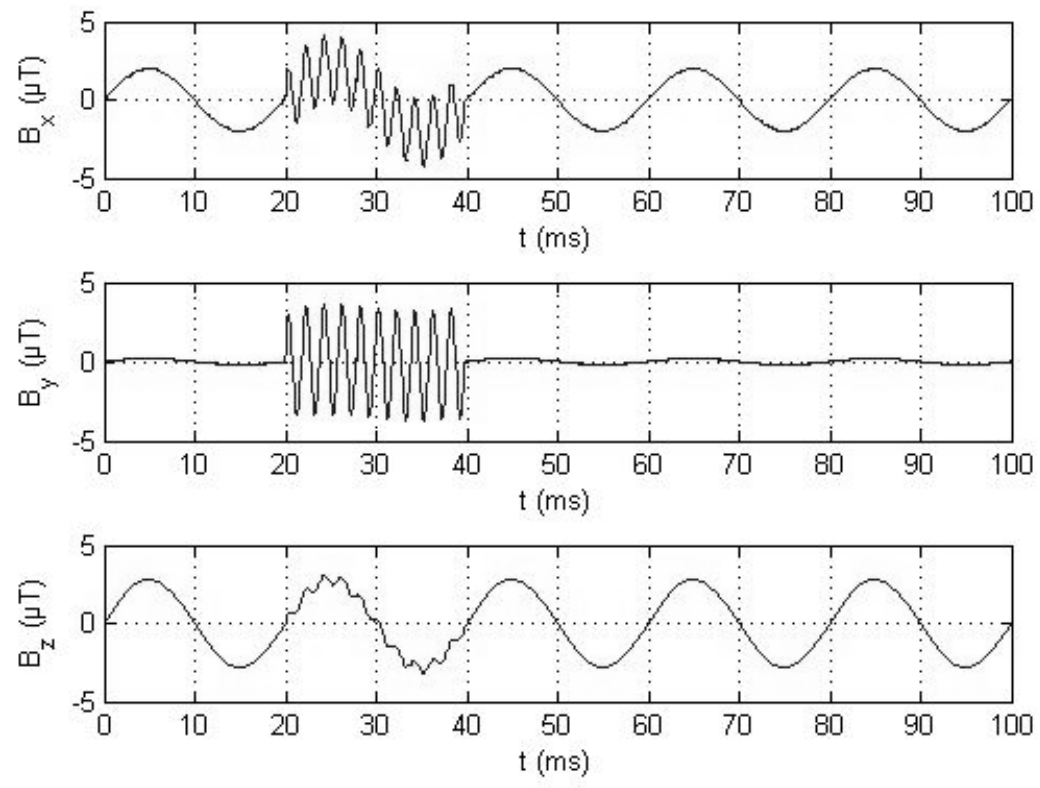

Fig. 4 Superimposed magnetic fields of a welding device and a line current

From one eigenvalue of zero $\left(\lambda_{1}=0.0\right)$ and two eigenvalues distinct from zero $\left(\lambda_{2}=1.1845\right.$, $\lambda_{3}=6.2861$ ) it is possible to infer that a superimposition of two sources took place. The ICA algorithm provides the time series depicted in Fig. 5. 

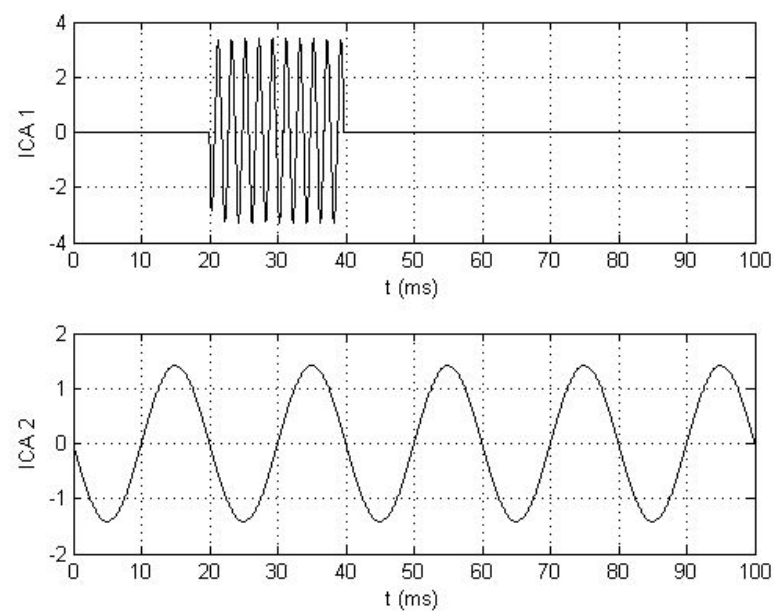

Fig. 5 Independent Components calculated with fast ICA

The independent components accord to the source time series. An Adjustment calculus with measured data and independent components allows to reconstruct the source time series and the direction vectors of the magnetic flux density of each source.

\section{Results and Discussion}

The introduced methods can be used to handle three-dimensional measurement data. Especially the application on magnetic vectorfields can facilitate their evaluation.

The observation of the eigenvalues of time samples allow the analysis of measurement data while measuring. This observation results in additional information about sources in the measured data. The separation of superimposed sources enables an evaluation of each source and a spatial filtering to fade out disturbing sources during the measurement. Especially when a superimposition of sources is measured the ICA algorithm offers an opportunity to separate fields from each other.

The new possibility to detect a superimposition of two field sources under certain conditions emerged out of exemplary data. Superimposed fields have been separated from each other by means of ICA. This method can help to evaluate each source distinctly - especially when pulsed fields are measured. The introduced methods will be implemented in terms of new measurement equipment.

\section{References}

[1] Guidelines for Limiting Exposure to Time Varying Electric, Magnetic and Electromagnetic Fields (Up to 300 GHz), ICNIRP Guidelines, Health Physics, 1998 Vol. 74 No 4

[2] J.P. Reilly, Peripheral Nerve Stimulation by Induced Electric Currents: Exposure to Time-Varying Magnetic Fields, Medical \& Biological Engineering \& Computing, 1989, Vol. 27 (2), pp. 101-110

[3] Eichhorn, K.Fr, Bewertung breitbandiger niederfrequenter Feldexpositionen insbesondere durch gepulste Felder; Elektromagnetische Verträglichkeit EMV 2008, VDE Verlag, 2008

[4] Guidence on Determining Compliance of Exposure to Pulsed and Coplex Non-Sinusoidal Waveforms Below $100 \mathrm{kHz}$ With ICNIRP Guidelines, Health Physics, 2003, vol. 84, No.3, pp.383-387

[5] T.R. Knösche, E.M. Berends, H.R.A. Jagers and M.J. Peters, Determining the Number of Independent Sources of the EEG: A Simulation Study on Information Criteria, Brain Topography, 1998, Vol. 11 (2), pp.111-124

[6] X. Bai and B. He, Estimation of Number of Independent Brain Electric Sources From the Scalp EEGs, IEEE Transactions on Biomedical Engineering, 2006, vol. 53, No 10, pp. 1883-1892

[7] P. Common, Independent Component Analysis, A New Concept?, Signal Processing, 1994 (36), pp. 287314.

[8] A. Hyvärinen and E. Oja, Independent Component Analysis: Algorithms and Applications, Neural Networks, 2000, 13(4-5), pp. 411-430

[9] C. Rückerl, K. Fr. Eichhorn, H. Bauer: Zur quellenorientierten Bewertung überlagerter magnetischer Felder; Elektromagnetische Verträglichkeit EMV 2008, VDE Verlag 2008 